\section{Congenital disorders of glycosylation caused by defects in mannose addition during $\mathbf{N}$-linked oligosaccharide assembly}

\author{
Peter Orlean
}

Department of Biochemistry, University of Illinois-Urbana-Champaign, 309 Roger Adams Laboratory, 600 South Mathews Avenue, Urbana, Illinois 61801, USA. Phone: (217) 333-4139; Fax: (217) 244-5858; E-mail: p-orlean@uiuc.edu.

Proteins that traverse the secretory pathway of eukaryotic cells can be covalently modified with carbohydrates, which are important for their stability and folding, and which mediate diverse recognition events in growth and development (1). Defects in the attachment of carbohydrate to protein give rise to mental and psychomotor retardation, dysmorphism, and blood coagulation defects $(2,3)$. These symptoms, referred to as CDG (for congenital disorders of glycosylation, or, until recently, for carbohydrate-deficient glycoprotein syndrome), are caused by mutations that affect the pathway for N-glycosylation.

In this pathway (Figure 1), a branched saccharide of 14 sugars is built up on the polyisoprenoid carrier lipid dolichyl pyrophosphate (PP-Dol), then transferred to asparagines in the sequence Asn-X-Ser/Thr (where $\mathrm{X}$ is any amino acid except proline). Synthesis of the lipid-linked oligosaccharide (LLO) is carried out by enzymes in the membrane of the endoplasmic reticulum (ER) that act in a specific order, each transferring a specific sugar to the nascent glycolipid $(4,5)$. Two $N$-acetylglucosamines (Glc-
NAc's) and 5 mannoses (Man) are transferred from the sugar nucleotides UDPGlcNAc and GDP-Man at the cytoplasmic face of the membrane, after which the $\mathrm{Man}_{5} \mathrm{GlcNAc}_{2}-P P$-Dol formed is flipped into the lumen of the ER, where 4 mannoses and 3 glucoses are added from dolichol-phosphate-mannose (Dol-P-Man) and Dol-P-glucose. The oligosaccharide is then attached to protein by oligosaccharyltransferase, a complex of at least 8 proteins (6). After transfer, the 3 glucoses and 1 mannose are trimmed from the oligosaccharide, and once the glycoprotein is transported to the Golgi apparatus, further mannoses can be removed and other sugars added to the residual oligosaccharide, generating diverse structures.

Many steps in $\mathrm{Glc}_{3} \mathrm{Man}_{9} \mathrm{GlcNAc}_{2}-\mathrm{PP}$ Dol assembly have been defined genetically using yeast asparagine-linked glycosylation (alg) mutants, an approach pioneered by Huffaker and Robbins (7). Mutations in LLO assembly lead to formation of incomplete oligosaccharides, which are poor oligosaccharyltransferase substrates; asparagines that normally are glycosylated may remain unmodified, yielding glycoproteins with fewer carbohydrate chains. In the diagnosis of CDG, hypoglycosylation is inferred from altered isoelectric focusing patterns of transferrin (2).

The CDGs defined so far can be divided into 2 types, depending on whether they impair LLO assembly and transfer (CDG-I) or whether they affect trimming of the protein-bound oligosaccharide or the addition of sugars to it (CDG-II). (A new nomenclature for CDGs has been proposed by the participants in the First International Workshop on CDGs [Leuven, Belgium, November 12-13, 1999] and is followed here.) Two of the type I CDGs arise from deficiencies in the supply of mannose for glycosyl transfer reactions because of defects in the formation of Man-6-P from fructose-6-P by phosphomannose isomerase (PMI) (CDG-Ib, formerly Type $\mathrm{Ib}$ ) or in the conversion of Man-6P to Man-1-P by phosphomannomutase (PMM) (CDG-Ia, formerly Type Ia) (3). A third CDG-I (CDG-Ic, originally Type $\mathrm{V})$ is caused by a defect in the transfer of glucose to $\mathrm{Man}_{9} \mathrm{GlcNAc}_{2}$-PP-Dol by the hAlg6 protein $(8,9)$.

\section{Figure 1}

Assembly of the dolichol-linked precursor in N-glycosylation and steps affected in type I CDG. The Man ${ }_{5} \mathrm{GlcNAc}_{2}$-PP-Dol intermediate is synthesized on the cytoplasmic side of the ER membrane by the successive transfer of GlcNAc and mannose from UDP-GlcNAc and GDP-Man. The Dol-PP-heptasaccharide is then translocated across the ER membrane. Four more mannoses and then 3 glucoses are added from DolP-Man and Dol-P-glucose, after which the oligosaccharide is transferred to certain asparagines in protein by oligosaccharyltransferase (OT-ase). Shown are the steps in the formation of GDP-Man and Dol-P-Man and the other glycosylation pathways in which Dol-P-Man can serve as donor. The steps blocked by the known type I CDGs and the genes affected are indicated. F-6-P, fructose-6-P; M, mannose; G, glucose; $\mathrm{GN}$, glucosamine; PI, phosphatidylinositol.

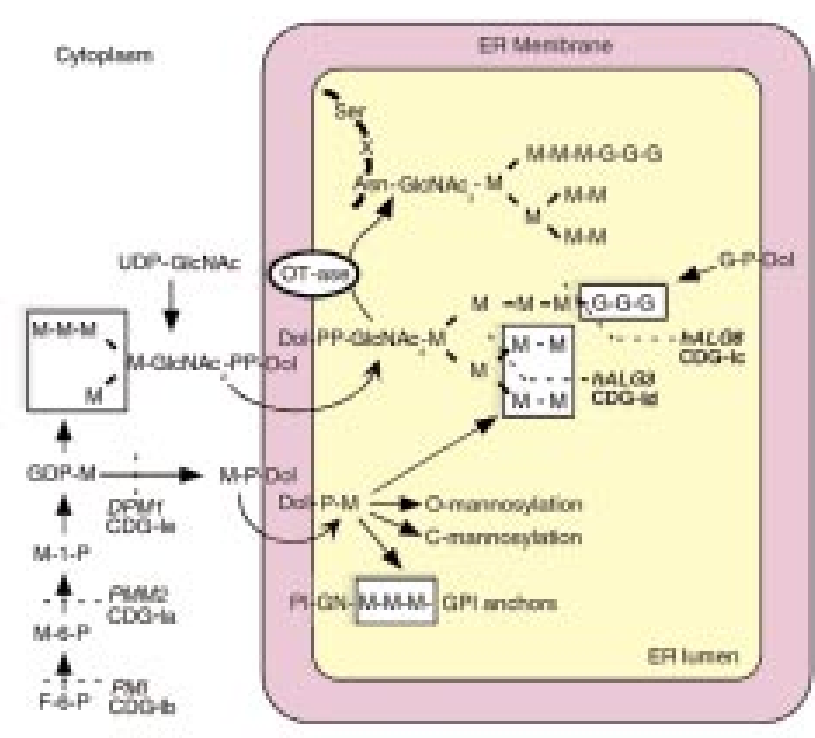


Two reports in this issue $(10,11)$ and 1 elsewhere (12) describe new CDGs, types Id and Ie, in which LLO assembly is blocked at $\mathrm{Man}_{5} \mathrm{GlcNAc}_{2}-P P$-Dol. Fibroblasts from CDG-Ie patients (10, 11) have low activity of Dol-P-Man synthase, which transfers mannose from GDP-Man to Dol-P to generate the donor of the luminally added mannoses. The human enzyme has 3 subunits $(13,14)$. Dpm1 is catalytic (15). Dpm 2 and Dpm 3 are small proteins; Dpm2 promotes binding of Dol-P to the enzyme. CDG-Ie patients have a point mutation in Dpm 1 , and the mutant enzyme's $K_{\mathrm{m}}$ for GDP-Man is 6fold higher than normal. In CDG-Id cells, Dol-P-Man synthase is normal, and transfer of mannose from Dol-PMan to the LLO is affected by a missense mutation in the $h A L G 3$ gene (12).

Of the new CDGs, type Id specifically affects N-glycosylation. However, the Dol-P-Man synthase deficiency that leads to CDG-Ie can have further biochemical consequences: Dol-P-Man donates the mannoses found in glycosylphosphatidylinositol (GPI) membrane anchors, the mannose attached through C-linkage to certain tryptophans, and the mannose in O-linked glycoproteins (16-18). A defect in GPI assembly leads to the disease paroxysmal nocturnal hemoglobinuria (PNH; 16), but it is not clear whether deficiencies in GPI synthesis or in O- or C-mannosylation contribute to the pathology of CDG-Ie; these patients apparently do not exhibit PNH symptoms, and the clinical features of CDG-Id and -Ie are similar. However, CDG-I cells always retain residual activity of an affected enzyme, which presumably permits them to glycosylate proteins and attach GPI anchors to them above a threshold level necessary for viability. It is possible that when GDP-Man and Dol-P-Man are in limited supply, they are used more efficiently in other pathways, so that GPI anchoring occurs but N-glycosylation is incomplete (19).

Deficiencies in mannose-containing precursors in CDG-Ia, -Ib, and -Ie might be predicted to be bypassed if the cells are supplemented with mannose. Indeed, the LLO defect in PMI-defective (CDG-Ib) and PMM-defective (CDG-Ia) fibroblasts can be corrected by supplying mannose in their medium, but study results differ on whether this is possible in CDG-Ie cells. PMI deficiency can also be bypassed in the patient: oral mannose therapy has been used successfully to treat CDG-Ib (3).

Additional types of CDG-I could arise from defects at other steps in assembly of the LLO, in its translocation across the membrane, or in its transfer to asparagine. The challenge now is to determine the structures and biochemical activities of the enzymes involved, and the mechanism of $\mathrm{Man}_{5} \mathrm{GlCNAc}_{2}$ $P P$-Dol translocation. A further issue is whether CDG-I symptoms are due to hypoglycosylation of a few specific proteins. If so, the roles of these glycoproteins in, for example, the nervous system, are of great interest. Because the progress made in identifying the genes mutated in the CDG-I subtypes has relied on work done in yeast, these studies are an excellent example of how basic research with a model organism can help us understand human genetic disease.

1. Varki, A. 1993. Biological roles of oligosaccharides: all of the theories are correct. Glycobiology. 3:97-139.

2. Jaeken, J., Carchon, H., and Stibler, H. 1993. The carbohydrate-deficient glycoprotein syndromes: pre-Golgi and Golgi disorders? Glycobiology. 3:423-428.

3. Freeze, H.H., and Aebi, M. 1999. Molecular basis of carbohydrate-deficient glycoprotein syndromes type I with normal phosphomannomutase activity. Biochim. Biophys. Acta. 1455:167-178.

4. Kornfeld, R., and Kornfeld, S. 1985. Assembly of asparagine-linked oligosaccharides. Annu. Rev. Biochem. 54:631-664.

5. Burda, P., and Aebi, M. 1999. The dolichol pathway of N-glycosylation. Biochim. Biophys. Acta. 1426:239-257.

6. Knauer, R., and Lehle, L. 1999. The oligosaccharyltransferase complex from yeast. Biochim. Biophys. Acta. 1426:259-273.

7.Huffaker, T., and Robbins, P.W. 1983. Yeast mutants deficient in protein glycosylation. Proc. Natl. Acad. Sci. USA. 80:7466-7470.

8. Körner, C., et al. 1998. Carbohydrate-deficient glycoprotein syndrome type $\mathrm{V}$ : deficiency of dolichyl$P$-Glc:Man ${ }_{9} \mathrm{GlcNAc}_{2}-P P$-dolichyl glucosyltransferase. Proc. Natl. Acad. Sci. USA. 95:13200-13205.

9. Imbach, T., et al. 1999. A mutation in the human ortholog of the Saccharomyces cerevisiae ALG6 gene causes carbohydrate-deficient glycoprotein syndrome type-Ic. Proc. Natl. Acad. Sci. USA. 96:6982-6987.

10. Imbach, T, et al. 2000. Deficiency of dolicholphosphate-mannose synthase- 1 causes congenital disorder of glycosylation type Ie. J. Clin. Invest. 105:233-239.

11. Kim, S., et al. 2000. Dolichol phosphate mannose synthase (DPM1) mutations define congenital disorder of glycosylation Ie (CDG-Ie). J. Clin. Invest. 105:191-198.

12. Körner, C., et al. 1999. Carbohydrate-deficient glycoprotein syndrome type IV: deficiency of dolichyl-P-Man:Man ${ }_{5}$ GlcNAc $_{2}$-PP-dolichyl mannosyltransferase. EMBO J. 18:6816-6822.

13. Maeda, Y., Tomita, S., Watanabe, R., Ohishi, K. and Kinoshita, T. 1998. DPM2 regulates biosynthesis of dolichol phosphate-mannose in mammalian cells: correct subcellular localization and stabilization of DPM1, and binding of dolichol phosphate. EMBO J. 17:4920-4929.

14. Kinoshita, T., Tanaka, S., Hino, J., Kangawa, K. and Maeda, Y. 1999. Human dolichol phosphatemannose-synthase consists of three subunits, DPM1, DPM2, and DPM2. Glycobiology. 9:1104 (Abstr.).

15. Orlean, P., Albright, C., and Robbins, P.W. 1988. Cloning and sequencing of the yeast gene for dolichol phosphate mannose synthase, an essential protein. J. Biol. Chem. 263:17499-17507.

16. Takeda, J., and Kinoshita, T. 1995. GPI anchor biosynthesis. Trends Biochem. Sci. 20:367-371.

17. Doucey, M.A., Hess, D., Cacan, R., and Hofsteenge, J. 1998. Protein C-mannosylation is enzyme catalyzed and uses dolichyl-phosphatemannose as a precursor. Mol. Biol. Cell. 9:291-300.

18. Strahl-Bolsinger, S., Gentzsch, M., and Tanner, W. 1999. Protein $O$-mannosylation. Biochim. Biophys. Acta. 1426: 297-307.

19. Thomas, L.J., et al. 1991. Functional analysis of Tcell mutants defective in the biosynthesis of glycosylphosphatidylinositol anchor. Relative importance of glycosylphosphatidyl-inositol anchor versus $N$-linked glycosylation in T-cell activation. J. Biol. Chem. 266:23175-23184. 